\title{
Precauciones para la práctica quirúrgica segura en paciente COVID- 19 positivo
}

Precautions to the safe surgical practice on COVID-19 positive patient

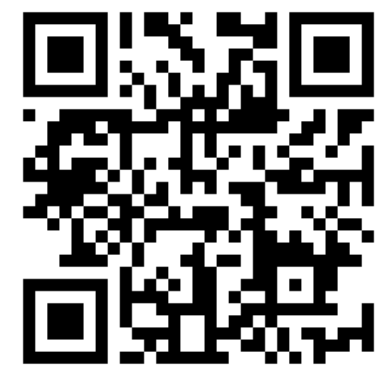

Recibido $16 / 04 / 2021$
${ }^{1}$ Dra. Iraís Martínez Daly Hospital de Guápiles, Limón, Costa Rica (iD) https://orcid.org/0000-0002-5985-9173

${ }^{2}$ Dra. María Angélica Montero Rojas Investigadora independiente, San José, Costa Rica

(iD) https://orcid.org/0000-0002-9736-8973

${ }^{3}$ Dra. Andrea Peña Montenegro Investigadora independiente, San José, Costa Rica

(i) https://orcid.org/0000-0002-7848-9059

Corregido $18 / 04 / 2021$
Aceptado 20/04/2021

\section{RESUMEN}

La situación actual ha sufrido grandes cambios generados por la pandemia causada por COVID-19. Conforme ha avanzado la pandemia, ha ido aumentando la cantidad de pacientes infectados y la posibilidad de que alguno de estos requiera cirugía. Por esta causa es de vital importancia que el cirujano conozca los aspectos básicos de la fisiopatología, tratamiento del SARS CoV-2 y las recomendaciones basadas en evidencia para disminuir el contagio durante la práctica quirúrgica. Se sabe que la manifestación clínica más frecuente de COVID-19 es fiebre, y su diagnóstico es preferiblemente realizado por PCR, la cual es beneficiosa previo a procedimientos quirúrgicos. Durante la actual pandemia se ha acordado entre las diferentes sociedades de cirujanos suspender las cirugías electivas, sin embargo, con tal cantidad de pacientes infectados se vuelve una prioridad la seguridad del paciente y de los profesionales implicados, para poco a poco, lograr la reintroducción de los procedimientos quirúrgicos electivos.

PALABRAS CLAVE: COVID-19, SARS CoV-2; cirugía; recomendaciones.

\section{ABSTRACT}

The current situation has suffered big changes due to the pandemic caused by COVID-19, which started in March of the past year. As the pandemic progresses the quantity of infected patients increases and the possibility that one of these patients would need surgery increases 
too. Which is why it is of vital importance that the surgeon knows the basic aspects of the pathophysiology of SARS CoV-2 and the recommendations based on evidence to reduce the contagion during the surgical practice. It is known that the most frequent clinical manifestation of COVID-19 is fever and the diagnosis is made preferably by PCR, which is beneficial previous to surgical procedures. During the pandemic it has been consensus between the different societies of surgeons to suspend elective surgery, however, with the big quantity of infected patients the security of the patient and the professionals involved in the surgical procedure becomes a priority, to, little by little achieve the reintroduction of elective surgery. In this article a review of the current recommendations to achieve this will be made.

\section{KEYWORDS: COVID-19; SARS CoV-2; surgery; recommendations.}

\footnotetext{
${ }^{1}$ Médica general, graduada de la Ciencias Médicas (UCIMED), Cód. MED16569. Correo: iramarti13@hotmail.com

${ }^{2}$ Médica general, graduada de la Ciencias Médicas (UCIMED), Cód. MED16653. Correo: mari.montero.rojas@hotmail.com

${ }^{3}$ Médica general, graduada de la Ciencias Médicas (UCIMED). Cód. MED16544. Correo: anvepea@hotmail.com
}

\section{INTRODUCCIÓN}

La situación actual está marcada por el cambio generado por la pandemia producida por el Coronavirus 2019 (COVID-19), a la enfermedad causada por éste se le llamó Síndrome Respiratorio Agudo Severo Coronavirus 2 (SARS CoV-2). Esta, inició en diciembre 2019, cómo una neumonía de etiología desconocida en Wuhan, China, que se identificó por primera vez en enero 2020 y la Organización Mundial de la Salud (OMS) en marzo de dicho año declaró en estado de pandemia $(1,2)$.

La OMS reporta que a nivel global se han confirmado más de 127 millones de casos, incluyendo dentro de estos más de dos millones y medio de muertes por dicha causa (3). Esta situación ha sido un reto para los sistemas de salud, llevando a la saturación de algunos centros y redistribución de recursos. Esto ha tenido repercusiones, sobre todo en las especialidades quirúrgicas, debido al cierre de quirófanos, suspensión de intervenciones electivas y redistribución del personal médico $\mathrm{y}$ quirúrgico $(2,4)$.
Debido a esta gran cantidad de pacientes infectados, se ha vuelto una posibilidad que alguno requiera una intervención quirúrgica en dicho contexto $(2,4)$. Es aceptado que en pacientes COVID-19 se posponga la cirugía en la medida de lo posible y se reconsidere la indicación de dicha intervención; ya que, si hay alguna alternativa no quirúrgica esta se prefiere, al menos mientras el paciente siga con infección activa, ya que algunos autores han documentado aumento de morbimortalidad quirúrgica en estos casos $(4,5)$.

Para el cirujano es importante conocer la presentación clínica, diagnóstico y tratamiento empleado. El objetivo del presente artículo es brindar una breve revisión bibliográfica de la enfermedad COVID 19 haciendo énfasis a las diferentes recomendaciones basadas en evidencia para disminuir el contagio del personal de salud y de los pacientes durante la práctica quirúrgica y lograr que esta sea segura (2).

\section{MÉTODO}

Se realizó una revisión bibliográfica utilizando varias plataformas, entre las que 
destaca, Cochrane, New England Journal of Medicine, Revista Scielo, lineamientos dados por el Ministerio de Salud de Costa Rica, y el sistema de reporte de casos de COVID-19 de la OMS, entre otros. Se revisaron múltiples artículos, todos citados en la bibliografía, los cuales se utilizaron cómo guía bibliográfica para la realización de este artículo. El intervalo de tiempo para la búsqueda fue los dos últimos años.

\section{GENERALIDADES}

Desde su inicio el COVID-19 se ha propagado rápidamente, inició cómo una zoonosis (6), sin embargo, se ha demostrado transmisión altamente efectiva de persona-persona, a través de contacto directo, aerosoles, por medio de gotas, fómites y potencialmente por contacto fecaloral $(7,8,9,10)$. Un estudio realizado en el Hospital de Jinyintan y el Hospital Pulmonar de Wuhan concluyó que hay factores de riesgo que implican mayor susceptibilidad al SARS CoV-2, estos incluyen asma, diabetes mellitus, enfermedad cardiovascular, cáncer, tabaquismo y obesidad (11). Además se identificaron factores de riesgo que pueden ayudar al médico o cirujano a identificar pacientes con mal pronóstico de manera temprana, se incluyen edad avanzada, SOFA Score elevado y Dímero-D mayor de $1 \mu \mathrm{g} / \mathrm{mL}$ (9).

\section{PRESENTACIÓN CLÍNICA}

Sus manifestaciones clínicas son leves en el $81 \%$ de los casos y un $1,2 \%$ son asintomáticos $(12,13)$. El síntoma más frecuente de COVID-19 es la fiebre, que ocurre en $87,9 \%$ de los pacientes, hay tos seca hasta en un $68 \%$ de los pacientes y fatiga, en un $38 \%$ de los casos, estos tres siendo los síntomas más frecuentes $(12,14)$.
Sintomatología menos frecuente incluye producción de esputo en un $28 \%$ y diarrea en un $3 \%$ (14). Aunque hay más síntomas de SARS CoV-2, no es el objetivo de este artículo nombrarlos.

\section{DIAGNÓSTICO Y TRATAMIENTO}

El diagnóstico de COVID-19 se realiza de preferencia mediante prueba de reacción en cadena de polimerasa en tiempo real (PCRTR) por medio de hisopado naso $u$ orofaríngeo y se realiza en los pacientes sospechosos de tener COVID-19 $(15,16)$.

Según los lineamientos del Ministerio de Salud, en Costa Rica un caso sospechoso es una persona que cumple con criterios clínicos y epidemiológicos, los cuales son, inicio súbita de fiebre y tos; o aparición de dos o más signos y síntomas (fiebre, tos debilidad general/fatiga, cefalea, mialgias, dolor de garganta, congestión nasal, anorexia/nauseas/vómitos, diarrea estado mental alterado) y además que cumpla con al menos uno de los siguientes criterios: (a) no tener otra causa que explique sus síntomas (b) historia de viaje fuera del país en los 14 días previos al inicio de síntomas (c) historial de haber frecuentado un distrito o cantón que se encuentre en alerta naranja en los 14 días previos al inicio de los síntomas (d) antecedente de contacto cercano, previo al inicio de los síntomas, con una persona que salió del país en los últimos 14 días o contacto con alguna persona que haya sido contacto directo de un caso confirmado. También se denomina sospechoso a una persona con infección respiratoria aguda grave (IRAG), con anosmia (pérdida del sentido del olfato) 0 disgeusia reciente (cambio en la percepción del gusto) sin otros etiologías que expliquen la presentación clínica, una persona con enfermedad respiratoria aguda de cualquier 
grado de severidad, que dentro de los 14 días anteriores al inicio de la enfermedad tuvo contacto físico cercano con un caso confirmado, probable, sospechoso o visito/laboró en un centro médico público o privado (17).

Para el diagnóstico también se pueden utilizar pruebas serológicas, aunque, un resultado negativo durante los primeros siete días de enfermedad no puede descartarla (18). En cuanto al tratamiento, es de soporte y antiinflamatorio principalmente. Se ha propuesto el uso de fármacos antivirales cómo lopinavir / ritonavir, y otros cómo inhibidores de la síntesis de ADN, cloroquina, y péptido basado en la enzima convertidora de angiotensina, ninguno de estos comprobado cómo tratamiento efectivo $(8,19)$. El uso de glucocorticoides en múltiples estudios clínicos se ha documentado útil para el manejo de neumonía ya sea por SARS, MERS o incluso neumonía por influenza, actualmente hay pobre evidencia que el beneficio de los glucocorticoides sea mayor que el riesgo de su uso, ya que se ha indicado que estos podrían retrasar la eliminación del virus y aumentar el riesgo de infección secundaria. El periodo de ventana para el uso de esteroides también es muy importante, ya que, pacientes cursando enfermedad grave usualmente cursan con deterioro abrupto a las 2 semanas del inicio de los síntomas, este tratamiento se utiliza solamente en pacientes con complicaciones severas por COVID-19 (20).

El uso de tomografía computarizada (TAC) es más eficaz que otros estudios para detectar cambios pulmonares por COVID-19 y tiene una sensibilidad del $91 \%$ y hasta $90 \%$ de los pacientes COVID-19 positivos tiene hallazgos inespecíficos en el TAC entre 3 y 5 días de sintomatología (21).

\section{RECOMENDACIONES PARA LA INTERVENCIÓN QUIRÚRGICA}

Varias asociaciones de cirujanos a nivel mundial han emitido recomendaciones $y$ guías clínicas para generalizar la práctica quirúrgica segura durante la situación vivida durante la pandemia por COVID-19 (7).

La Sociedad Americana de Endoscopía y Cirugía Gastrointestinal (SAGES, por sus siglas en inglés) y el Colegio Americano de Cirugía (ACS, igualmente por sus siglas en inglés) dan recomendaciones para racionalizar los servicios de cirugía; en estas se incluye (a) posponer todas las cirugías electivas, el manejo quirúrgico debe ser limitado sólo para cirugías de emergencia o pacientes con malignidad que podría progresar, (b) limitar al máximo el personal en la sala de operaciones, todo el personal no indispensable debe realizar teletrabajo o quedarse en casa $(22,23)$.

No obstante, estas recomendaciones fueron publicadas al inicio de la pandemia. Actualmente nos encontramos en un período de adaptación, dónde la prioridad para que, eventualmente se logre la reintroducción de las cirugías electivas debe ser la seguridad del paciente y de los profesionales implicados (13).

En cuanto a los pacientes oncológicos hay recomendaciones generales, cómo considerar manejo no quirúrgico cuando sea apropiado, al igual que en pacientes no oncológicos $(6,24)$. En pacientes con sospecha de COVID-19 se propone esperar el resultado del PCR por COVID-19, pero en ciertas situaciones se sabe que no hay disponibilidad inmediata ni tiempo para esperar el resultado, en dichos casos se podría considerar cómo opción realizar TAC preoperatorio cómo técnica de diagnóstico por imagen (24). Sin embargo, el uso de TAC cómo método de tamizaje o diagnóstico de 
COVID-19 no es recomendado por el Colegio Americano de Radiólogos, su indicación es utilizarlo en pacientes sintomáticos, hospitalizados y con indicaciones para el mismo. La toma de decisiones no debe basarse en este estudio, ya que un TAC normal no descarta enfermedad y si tiene hallazgos tampoco hace que eso sea específico de COVID-19 (21).

Por otro lado, se ha documentado que en la vía aérea alta de los pacientes COVID-19 asintomáticos se encuentran altas concentraciones de SARS CoV-2, esto implica que procedimientos en dicha área sean de alto riesgo de contagio por la generación de aerosoles. Durante la cirugía es importante considerar que hay procedimientos que pueden potenciar este proceso, y que el virus sea transmitido cómo pequeñas gotas en suspensión de gas $(2,25)$. Estos procedimientos de alto riesgo incluyen, intubación endotraqueal, ventilación no invasiva, traqueotomía, reanimación cardiopulmonar, ventilación manual, broncoscopías y endoscopías en general $(6,10,26,27)$. En estos casos se recomienda el uso de equipo de protección personal (EPP) preferiblemente N95 o el que dé mayor nivel de protección y previo a los mismos realizar PCR por COVID-19 del paciente asintomático de rutina $(1,23)$. De hecho, también los procedimientos que impliquen saliva, heces y sangre se consideran procedimientos de riesgo, ya que estos tienen potencial de presentar carga viral, aunque se desconoce si el virus es infeccioso en muestras extrapulmonares $(7,28)$.

\section{PREVIO A LA CIRUGÍA}

Según el ACS el uso de EPP se recomienda en todos los procedimientos realizados en paciente COVID-19 positivo o sospechoso, se debe utilizar mascarilla N95, escudo facial o careta y protección ocular con delantal protector y guantes. EI EPP posteriormente se quita y desecha de la manera correcta, antes y después se debe realizar lavado de manos. Además, durante la intubación del paciente todo el personal no necesario debe mantenerse fuera de la sala, por el riesgo de aerosoles. Se debe intentar que el procedimiento de intubación se logre en primera intención para disminuir la instrumentación repetida de la vía aérea (23).

De igual manera, se recomienda el uso de salas de operaciones con presión negativa, en caso de tener disponibilidad y siempre se debe utilizar la misma sala para los pacientes COVID-19 positivo durante la pandemia $(23,29)$. En caso de que sea posible se debe determinar si el paciente es COVID-19 positivo previo a la cirugía, si se determina que el paciente tiene infección por SARS CoV-2 se debe realizar una evaluación del riesgo hacia el paciente y hacia el personal de salud, usualmente conformado por un equipo multidisciplinario. (30) Siempre se deben de tomar todas las medidas de protección para el equipo (25).

\section{DURANTE LA CIRUGÍA}

SAGES y ACS recomiendan limitar la cantidad de personal presente durante la cirugía, como ya se mencionó previamente, y que se minimice el uso de los instrumentos de electrocirugía; en caso de que sean necesarios para la intervención, se deben utilizar al nivel más bajo posible para obtener el beneficio de estos y se debe asociar el uso de un evacuador de humo $(22,23,31)$.

Hay controversia en cuanto al abordaje que se prefiere, SAGES recomienda considerar el uso de abordaje laparoscópico con la 
salvedad que se realicen (a) incisiones lo más pequeñas posibles para el paso de los puertos laparoscópicos, (b) que la presión de insuflación con monóxido de carbono se mantenga al mínimo y, (c) que se utilice un sistema de evacuación de humo para evacuar el neumoperitoneo, antes de quitar el trocar, cerrar al paciente $o$ hacer conversión a procedimiento abierto (22). Además, se destacan los beneficios ya conocidos del abordaje laparoscópico, que incluyen disminución del tiempo de estancia hospitalaria y disminución de complicaciones en pacientes COVID-19 positivo, que potencialmente podrían complicarse más que un paciente no infectado $(22,28)$. Cómo se demostró en un estudio de cohortes realizado en Brescia, Italia, en el que se tomó en cuenta 41 pacientes COVID-19 positivo y 82 no infectados, a los que se les realizó cirugías de diferentes especialidades quirúrgicas, y se documentó que en los pacientes COVID19 positivo hubo diferencia significativa en cuánto a mortalidad temprana $\mathrm{y}$ complicaciones postoperatorias, y se documentó durante este estudio, que neumonía y complicaciones trombóticas estuvieron significativamente asociadas a COVID-19 (32). Por otro lado, el ACS recomienda que se evite el abordaje laparoscópico.

Aún no se ha dilucidado si el uso de abordaje laparoscópico produce mayor riesgo de transmisión del COVID-19, pero se sabe que, al liberarse el aire contenido en el neumoperitoneo, éste podría estar contaminado y aumentar el riesgo de contagio, no obstante, no hay datos para preferir la cirugía abierta (33). También se sabe que el uso de bisturí ultrasonográfico y de equipo eléctrico utilizado con el abordaje mínimamente invasivo puede producir gran cantidad de humo, y se ha descrito que cuando se utilizan estos instrumentos con baja temperatura no se inactivan efectivamente los componentes virales, de hecho, se ha detectado otros virus cómo Virus de Papiloma humano y VIH en humo quirúrgico, por lo que no se puede excluir el riesgo de contagio de COVID-19 a partir de este (33).

\section{POSTERIOR A LA CIRUGÍA}

El ACS recomienda que para el transporte del paciente COVID-19 luego de la cirugía, hacia recuperación o la unidad de cuidados intensivos ( $\mathrm{UCI})$, se debe minimizar al máximo el personal que participa del traslado y se debe mantener el uso de EPP, es importante recalcar que el EPP que se utilice, no debe ser el mismo que se usó durante la cirugía (23). En un protocolo realizado en Singapur, se indica que para los pacientes que no requieran ir a la $\mathrm{UCl}$ de manera postoperatoria, se puede mantener al paciente en la sala de operaciones hasta que se recupere por completo, sin embargo, esto es poco práctico. Cuando se movilice al paciente se debe despejar por completo la zona y también dónde se va a hospitalizar. Según este protocolo, además de quitarse el EPP adecuadamente, todo el equipo quirúrgico debe bañarse antes de reanudar sus tareas, y luego su uso en casos confirmados por COVID-19 se puede utilizar vaporizador con peróxido de hidrógeno en la sala de operaciones (33).

\section{POST OPERATORIO DE PACIENTES COVID-19 POSITIVOS}

En un estudio retrospectivo realizado en Wuhan, China se analizaron pacientes a los que se les realizó cirugía abdominal de emergencia por un periodo de un mes, de estos 8 eran COVID-19 positivos y 22 no 
estaban infectados, se comparó los resultados, y se documentó que en pacientes con infección leve o asintomáticos la recuperación post operatoria no se ve afectada por el virus. Sin embargo 4 de los 8 pacientes COVID-19 positivos presentaron complicaciones y se demostró mortalidad relativamente alta luego de la cirugía de emergencia (5). Usualmente se observan complicaciones post operatorias sobre todo en pacientes COVID-19 adultos mayores y con comorbilidades (34).

\section{CONCLUSIONES}

La pandemia producida por el SARS CoV-2 ha constituido un reto para los sistemas de salud y los médicos que trabajan en estos, es importante que, los cirujanos y el personal en general conozca los aspectos básicos del diagnóstico y manejo de COVID-19, y además tengan claras las recomendaciones actuales para la realización de procedimientos operatorios, ésta es la mejor manera de lograr una práctica quirúrgica segura, tanto para el paciente COVID-19 positivo cómo para el personal de salud.

\section{Los autores declaran no tener conflicto de interés.}

\section{REFERENCIAS}

1. Zizzo M, Bollino $R$, Castro Ruiz C, Biolchini F, Bonilauri S, Sergi W, et al. Surgical management of suspected or confirmed SARS-CoV-2 (COVID19)-positive patients: a model stemming from the experience at Level III Hospital in EmiliaRomagna, Italy. European Journal of Trauma and Emergency Surgery [Internet]. 2020 [citado el 25 de Febrero 2021]; 46(3):513-517. Disponible: https://link.springer.com/article/10.1007\%2Fs000 68-020-01377-2

2. García-Novoa A, Casal-Beloy I. Crisis sanitaria COVID-19: el papel de un cirujano. Revista de Cirugía Española [Internet]. 2020 [citado el 25 de Febrero 2021];98(7):420-421. Disponible en: https://www.ncbi.nlm.nih.gov/pmc/articles/PMC71 $\underline{41444 /}$
3. COVID Response Fund, World Health Organization. WHO Coronavirus Disease (COVID-19) Dashboard [Internet]. World Health Organization. 2020 [citado el 2 de Marzo 2021]. Disponible en: https://covid19.who.int

4. Balibrea J, Badia J, Rubio Pérez I, Martín Antona E, Álvarez Peña E, García Botella S, et al. Manejo quirúrgico de pacientes con infección por COVID19. Recomendaciones de la Asociación Española de Cirujanos. Cirugía Española [Internet]. 2020 [citado el 22 de Febrero 2021]; 98(5):251-259. Disponible

en: https://www.ncbi.nlm.nih.gov/pmc/articles/PMC72 70428/

5. Cai M, Wang G, Zhang L, Gao J, Xia Z, Zhang P, et al. Performing abdominal surgery during the COVID-19 epidemic in Wuhan, China: a singlecentred, retrospective, observational study. British Journal of Surgery [Internet]. 2020 [citado el 24 de Febrero 2021]; 107(7):e183-e185. Disponible en: https://bjssjournals.onlinelibrary.wiley.com/doi/full /10.1002/bjs.11643

6. Glibbery N, Karamali K, Walker C, O'Connor I, Fish B, Irune E. Tracheostomy in the coronavirus disease 2019 patient: evaluating feasibility, challenges and early outcomes of the 14-day guidance. The Journal of Laryngology \& Otology [Internet]. 2020 [citado el 22 de Febrero 2021];:1$17 . \quad$ Disponible en: https://www.cambridge.org/core/journals/journalof-laryngology-and-otology/article/tracheostomyin-the-coronavirus-disease-2019-patientevaluating-feasibility-challenges-and-earlyoutcomes-of-the-14dayguidance/9201F761C150099DBEA5F626F54D2 B3F

7. Rubio-Pérez I, Badia J, Mora-Rillo M, Martín Quirós A, García Rodríguez J, Balibrea J, et al. COVID-19: Conceptos clave para el cirujano. Cirugía Española [Internet]. 2020 [citado el 25 de Febrero 2021]; 98(6):310-319. Disponible en: https://doi.org/10.1016/j.ciresp.2020.04.009

8. Lai C, Shih T, Ko W, Tang H, Hsueh P. Severe acute respiratory syndrome coronavirus 2 (SARSCoV-2) and coronavirus disease-2019 (COVID19): The epidemic and the challenges. International Journal of Antimicrobial Agents [Internet]. 2020 [citado el 22 de Febrero 2021]; 55(3):105924. Disponible en: https://www.sciencedirect.com/science/article/pii/ S0924857920300674?via\%3Dihub

9. Zhou F, Yu T, Du R, Fan G, Liu Y, Liu Z, et al. Clinical course and risk factors for mortality of adult inpatients with COVID-19 in Wuhan, China: 
a retrospective cohort study. The Lancet [Internet]. 2020 [citado el 21 de Febrero 2021]; 395(10229):1054-1062. Disponible en: https://www.thelancet.com/journals/lancet/article/ PIIS0140-6736(20)30566

10. Heffernan D, Evans H, Huston J, Claridge J, Blake $D$, May $A$, et al. Surgical Infection Society Guidance for Operative and Peri-Operative Care of Adult Patients Infected by the Severe Acute Respiratory Syndrome Coronavirus-2 (SARSCoV-2). Surgical Infections [Internet]. 2020 [citado el 23 de Febrero 2021];21(4):301-308. Disponible en: https://doi.org/10.1089/sur.2020.101

11. Guan W, Ni Z, Hu Y, Liang W, Ou C, He J, et al. Clinical Characteristics of Coronavirus Disease 2019 in China. New England Journal of Medicine [Internet]. $2020 \quad$ [citado 23 Febrero 2021];382(18):1708-1720. Disponible en https://www.neim.org/doi/10.1056/NEJMoa20020 $\underline{32}$

12. Bchetnia M, Girard C, Duchaine C, Laprise C. The outbreak of the novel severe acute respiratory syndrome coronavirus 2 (SARS-CoV-2): A review of the current global status. Journal of Infection and Public Health [Internet]. 2020 [citado el 25 de Febrero 2021] ;:2-10. Disponible en: https://www.sciencedirect.com/science/article/pii/ S1876034120305918?

13. Gracia M, Rius M, Carmona F. Cirugía laparoscópica en tiempos de COVID-19. Clínica e Investigación en Ginecología y Obstetricia [Internet]. 2020 [Citado el 23 febrero 2021];47(3):106-110. Disponible en: https://www.sciencedirect.com/science/article/pii/ S0210573X20300484?via\%3Dihub

14. Jin Y, Yang H, Ji W, Wu W, Chen S, Zhang W, et al. Virology, Epidemiology, Pathogenesis, and Control of COVID-19. Viruses [Internet]. 2020 [citado el 22 de 2021];12(4):372. Disponible en: https://www.mdpi.com/1999-4915/12/4/372

15. Sociedad Española de Otorrinolaringología y Cirugía de Cabeza y Cuello. ¿Qué pruebas se realizan para diagnosticar la enfermedad de COVID-19? - SEORL-CCC [Internet]. Seorl.net. 2020 [citado el 23 de Febrero 2021]. Disponible en: https://seorl.net/pruebas-diagnostico-covid$\underline{19 /}$

16. Internet $\mathrm{H}$. Información general y lineamientos nacionales sobre la enfermedad por coronavirus (COVID-19) [Internet]. Inciensa.sa.cr. 2020 [citado el 24 Febrero 2021]. Available from: https://www.inciensa.sa.cr/actualidad/noticias/lnf ormacion general $\mathrm{y}$ lineamientos nacionales $\mathrm{s}$ obre la\%20enfermedad por coronavirus(COVID -19).aspx

17. Arroba Tijerino R, Torres Moreno A, Salguero Mendoza $C$, Delgado Jiménez $S$, Agüero Zumbado A, Mora Carvajal J, et al. Lineamientos Nacionales para Vigilancia de la enfermedad COVID-19 [Internet]. 18th ed. San José, Costa Rica:Ministerio de Salud de Costa Rica; 2021[citado el 10 de Abril 2021]. Disponible en: https://www.ministeriodesalud.go.cr/sobre minist erio/prensa/docs/LS VS 001 version 18 vigilan cia 08032021.pdf

18. Organización Mundial de la Salud y Organización Panamericana de la Salud; Directrices de laboratorio para la detección y el diagnóstico de la infección por el virus responsable de la COVID-19. [Internet]. 2020 [citado 24 de Febrero 2021]. Disponible en

https://iris.paho.org/bitstream/handle/10665.2/52 471/OPSIMSPHECOVID-

19200038 spa.pdf? sequence $=1$ \&isAllowed $=y$

19. Rahman S, Bahar T. COVID-19: The New Threat, Int J Infect. $2020 ; 7(1): \mathrm{e} 102184$. doi: 10.5812/iji.102184.

https://dx.doi.org/10.5812/iji.102184

20. Wen Z, Yan Z, Fengchun Z, Qian W, Taisheng L, Zhengyin $L$, et al. The use of anti-inflammatory drugs in the treatment of people with severe coronavirus disease 2019 (COVID-19): The Perspectives of clinical immunologists from China [Internet]. US National Library of Medicine National Institutes of Health. 2020 [citado el 3 de Marzo 2021]. Disponible en: https://www.ncbi.nlm.nih.gov/pmc/articles/PMC71 02614

21. Arbeláez Salgado M, Cadavid Congote A, Flórez Filomeno D, Garcés Otero J, Gómez Machado A, Guzmán Arango C,et al. Temas de interés para el cirujano. Revista Colombiana de Cirugía [Internet]. 2020 [Citado 23 Febrero 2021];35(2):154-160. Disponible en: https://www.revistacirugia.org/index.php/cirugia/a rticle/view/612

22. Pryor A. SAGES and EAES Recommendations Regarding Surgical Response to COVID-19 Crisis - SAGES [Internet]. SAGES. 2020 [citado el 25 de Febrero 2021]. Disponible en: https://www.sages.org/recommendationssurgical-response-covid-19/

23. American College of Surgeons. COVID-19: Consideraciones para la protección óptima de los cirujanos antes, durante y después de los procedimientos [Internet]. 2020 [citado el 24 de Febrero 2021]. Disponible: 
https://www.facs.org/covid-19/clinicalguidance/surgeon-protection/proteccion-optima

24. Pacheco Trujillo M, Torres Mesa $P$, Arias Amezquita F, Raúl Eduardo P, Mario Arturo A, Villarreal $\mathrm{R}$ et al. Recomendaciones para cirugía en pacientes con patologías oncológicas durante la pandemia COVID-19. Revista Colombiana de Cirugía [Internet]. 2020 [Citado el 22 Febrero 2021];35(2):162-170. Disponible en: https://www.revistacirugia.org/index.php/cirugia/a rticle/view/616

25. Lewis S, Smith B, Akinboyo I, Seidelman J, Wolfe C, Kirk A, et al. Early Experience with Universal Pre-procedural Testing for SARS-CoV-2 in a Relatively Low-Prevalence Area. Infection Control \& Hospital Epidemiology [Internet]. 2020 [citado el 25 de Febrero 2021];:1-9. Disponible en: https://www.cambridge.org/core/journals/infection -control-and-hospital-epidemiology/article/earlyexperience-with-universal-preprocedural-testingfor-sarscov2-in-a-relativelylowprevalencearea/51F0D9E350144904E0CE5D 8FEFE5AC82

26. Ministerio de Salud, Lineamientos generales para el uso del Equipo de Protección Personal (EPP), para prevenir la exposición al COVID-19 en servicios de salud y Centros de trabajo. [Internet]. 7th ed. San José, Costa Rica; 2020 [citado el 24 Febrero 2021]. Disponible en: https://www.ministeriodesalud.go.cr/sobre minist erio/prensa/docs/lg ss 006 EPP 30062020 v7. pdf

27. Cuevas-López L, Ayala J, Velásquez-Jiménez O, Navarro-Alean J, González-Higuera L, Zurita N, et al . Recomendaciones para el manejo de los pacientes quirúrgicos urgentes durante la pandemia COVID-19. rev. colomb. cir. [Internet]. 2020 June [citado el 22 de Febrero 2021] ; 35( 2 ): 143-152. Disponible en: http://www.scielo.org.co/scielo.php?script=sci_art text\&pid=S2011-75822020000200143\&Ing=en. http://dx.doi.org/10.30944/20117582.619

28. Cabrera-Vargas L, Pedraza Ciro M, Torregrosa Almonacid L, Figueredo E. Cirugía durante la pandemia del sars-cov-2 / covid-19: el efecto de la generación de aerosoles de partículas en escenarios quirúrgicos. Revista Colombiana de Cirugía [Internet]. 2020 [Citado el 25 February 2021];35(2):190-199. Disponible en: https://www.revistacirugia.org/index.php/cirugia/a rticle/view/625

29. Ti L, Ang L, Foong T, Ng B. What we do when a COVID-19 patient needs an operation: operating room preparation and guidance. Canadian Journal of Anesthesia/Journal canadien d'anesthésie [Internet]. 2020 [citado 24 de Febrero 2021];67(6):756-758. Disponible en: https://bjssjournals.onlinelibrary.wiley.com/doi/full /10.1002/bjs.11643

30. Givi B, Schiff B, Chinn S, Clayburgh D, lyer N, Jalisi $S$, et al. Safety Recommendations for Evaluation and Surgery of the Head and Neck During the COVID-19 Pandemic. JAMA Otolaryngology-Head \& Neck Surgery [Internet]. 2020 [citado el 24 de Febrero 2021];146(6):579. Disponible en: https://jamanetwork.com/journals/jamaotolaryngol ogy/fullarticle/2764032

31. Torregrosa Almonacid L, Prieto Ortiz R, CabreraVargas L, Ordoñez Mosquera Juliana María J, Sánchez Cortés E, Rodríguez Vargas $\mathrm{C}$ et al. Recomendaciones generales para los Servicios de Cirugía en Colombia durante la pandemia COVID-19 (SARS-CoV-2). Revista Colombiana de Cirugía [Internet]. 2020 [Citado el 22 Febrero 2021];35(2):265-281. Disponible en : https://www.revistacirugia.org/index.php/cirugia/a rticle/view/633

32. Doglietto F, Vezzoli M, Gheza F, Lussardi G, Domenicucci $M$, Vecchiarelli $L$ et al. Factors Associated With Surgical Mortality and Complications Among Patients With and Without Coronavirus Disease 2019 (COVID-19) in Italy. JAMA Surgery [Internet]. 2020 [Citado 24 de Febrero 2021];. Disponible en: https://jamanetwork.com/journals/jamasurgery/full article/2767370?resultClick=1

33. Hua Zheng $M$, Boni L, Fingerhut $A$. Annals of Surgery. Minimally invasive surgery and the novel coronavirus outbreak: lessons learned in China and Italy. [Internet]. Journals.Iww.com. 2020 [citado el 24 de Febrero 2021]. Disponible en: https://journals.Iww.com/annalsofsurgery/Docum ents/Minimally $\% 20$ invasive $\% 20$ surgery $\% 20$ and $\%$ 20the $\% 20$ novel $\% 20$ coronavirus $\% 20$ outbreak $\% 20$ $=$ $\%$ 20lessons $\% 20$ learned $\% 20$ in $\% 20$ China $\% 20$ and \%20ltaly.pdf

34. Alvarez-Bobadilla G, Domínguez-Cherit G, Acosta-Nava V, Guizar-Rangel M, Guido-Guerra $\mathrm{R}$, Garduño-López A. Manejo perioperatorio del paciente con COVID-19. Revista Mexicana de Anestesiología [Internet]. 2020 [citado el 24 de febrero 2021];43(2):35-46. Disponible en: https://www.medigraphic.com/pdfs/rma/cma2020/cma202f.pdf 